\title{
Identification of Novel Mitochondrial Pyruvate Carrier Inhibitors by Homology Modeling and Pharmacophore-Based Virtual Screening
}

\author{
Lamees Hegazy ${ }^{1,2, *}$, Lauren E. Gill ${ }^{3}$, Kelly D. Pyles ${ }^{3}$, Christopher Kaiho ${ }^{1}$, Sophia Kchouk ${ }^{1}$, Brian N. Finck ${ }^{4}$ (D), \\ Kyle S. McCommis ${ }^{3, * \mathbb{D}}$ and Bahaa Elgendy ${ }^{1,2,5}$ (D)
}

1 Center for Clinical Pharmacology, Washington University School of Medicine, University of Health Sciences \& Pharmacy, St. Louis, MO 63110, USA; Christopher.kaiho@uhsp.edu (C.K.); sophia.kchouk@uhsp.edu (S.K.); belgendy@wustl.edu (B.E.)

2 Department of Pharmaceutical and Administrative Sciences, University of Health Sciences \& Pharmacy, St. Louis, MO 63110, USA

3 Department of Biochemistry \& Molecular Biology, Saint Louis University School of Medicine, St. Louis, MO 63104, USA; lauren.gill@slu.edu (L.E.G.); kelly.pyles@health.slu.edu (K.D.P.)

4 Department of Medicine, Washington University School of Medicine, St. Louis, MO 63110, USA; bfinck@wustl.edu

5 Chemistry Department, Faculty of Science, Benha University, Benha 13518, Egypt

* Correspondence: lamees.hegazy@uhsp.edu (L.H.); kyle.mccommis@health.slu.edu (K.S.M.)

Citation: Hegazy, L.; Gill, L.E.; Pyles, K.D.; Kaiho, C.; Kchouk, S.; Finck, B.N.; McCommis, K.S.; Elgendy, B. Identification of Novel Mitochondrial Pyruvate Carrier Inhibitors by Homology Modeling and Pharmacophore-Based Virtual Screening. Biomedicines 2022, 10, 365. https: / / doi.org/10.3390/ biomedicines 10020365

Academic Editor: Maria Stefania Sinicropi

Received: 27 December 2021

Accepted: 31 January 2022

Published: 2 February 2022

Publisher's Note: MDPI stays neutral with regard to jurisdictional claims in published maps and institutional affiliations.

Copyright: (c) 2022 by the authors. Licensee MDPI, Basel, Switzerland. This article is an open access article distributed under the terms and conditions of the Creative Commons Attribution (CC BY) license (https:// creativecommons.org/licenses/by/ $4.0 /)$.

\begin{abstract}
The mitochondrial pyruvate carrier (MPC) is an inner-mitochondrial membrane protein complex that has emerged as a drug target for treating a variety of human conditions. A heterodimer of two proteins, MPC1 and MPC2, comprises the functional MPC complex in higher organisms; however, the structure of this complex, including the critical residues that mediate binding of pyruvate and inhibitors, remain to be determined. Using homology modeling, we identified a putative substrate-binding cavity in the MPC dimer. Three amino acid residues (Phe66 (MPC1) and Asn100 and Lys49 (MPC2)) were validated by mutagenesis experiments to be important for substrate and inhibitor binding. Using this information, we developed a pharmacophore model and then performed a virtual screen of a chemical library. We identified five new non-indole MPC inhibitors, four with $\mathrm{IC}_{50}$ values in the nanomolar range that were up to 7 -fold more potent than the canonical inhibitor UK-5099. These novel compounds possess drug-like properties and complied with Lipinski's Rule of Five. They are predicted to have good aqueous solubility, oral bioavailability, and metabolic stability. Collectively, these studies provide important information about the structure-function relationships of the MPC complex and for future drug discovery efforts targeting the MPC.
\end{abstract}

Keywords: mitochondrial pyruvate carrier; homology modeling; mutagenesis; pharmacophore modeling; virtual screening

\section{Introduction}

Mitochondria convert chemical energy stored in nutrients to adenosine triphosphate (ATP) and other forms of energy and play a number of important roles in intermediary metabolism. Pyruvate is used by the mitochondrion for oxidative metabolism by pyruvate dehydrogenase and is also carboxylated to provide anaplerotic substrates for the tricarboxylic acid (TCA) cycle. Transport of pyruvate across the inner mitochondrial membrane into the mitochondrial matrix is required for oxidation or carboxylation and is mediated by the mitochondrial pyruvate carrier (MPC) [1,2]. The MPC is a two-subunit carrier complex composed of MPC1 and MPC2 proteins and localized to the mitochondrial inner membrane (MIM) that works as a gatekeeper for pyruvate entry into mitochondria. These two subunits are paralogous proteins encoded by two genes that are highly conserved from yeast to humans. It is generally believed that MPC1 and MPC2 form a functional heterodimer; 
it has been shown that deletion of either MPC subunit leads to the destabilization and degradation of the other MPC protein [3,4]. Global genetic deletion of either MPC gene in mice is not compatible with life [3,4], likely due to developmental defects in the central nervous system and heart. However, conditional deletion in many tissues is well tolerated and can actually protect from the development of metabolic disease [5-7].

Development of specific and potent MPC modulators holds a great potential as therapeutics for myriad diseases including type 2 diabetes, nonalcoholic steatohepatitis (NASH), cancer, and even hair loss [8-11]. Known MPC inhibitors fall mainly into two categories: i. $\alpha$-cyanocinnamate derivatives (e.g., UK-5099) [12]; and ii. thiazolidinediones (TZDs) (e.g., rosiglitazone) [13], which lack selectivity, as most TZDs bind and activate the peroxisome proliferator-activated receptor-gamma (PPAR $\gamma$ ) (Figure 1). Compounds GW604714X and GW450863X are two potent thiazolidine inhibitors, which are comparable to UK-5099 in potency, but are also known to affect the activity of $\mathrm{K}_{\mathrm{ATP}}$ channels to inhibit potassium import [14].<smiles>N#C/C(=C\c1cn(-c2ccccc2)c2ccccc12)C(=O)O</smiles><smiles>CN(CCOc1ccc(CC2SC(=O)NC2=O)cc1)c1ccccn1</smiles>

Rosiglitazone<smiles>[Y4][R16](C)(C)O[Na]</smiles><smiles>COc1ccc(OC)c(/C=C2\SC(=O)NC2=O)c1</smiles>

Figure 1. Examples of known mitochondrial pyruvate carrier (MPC) inhibitors.

Despite widespread interest, development of specific and targeted MPC inhibitors is difficult due to the lack of structural information about the MPC complex. Additionally, lack of specific chemical probes for MPC hinders the ability to study the relationship between MPC phenotypic data and to launch the clinical translatability of this target. Herein, we identify key amino acids required for the binding of MPC inhibitors and report the identification of novel MPC inhibitors using a ligand-based pharmacophore modeling approach.

\section{Materials and Methods}

\subsection{Chemistry}

All starting materials were purchased from commercial suppliers and used without further purification. The purities of the final compounds were characterized by highperformance liquid chromatography (LC/MS) using a gradient elution program (Ascentis Express Peptide C18 column, acetonitrile/water 5/95/95/5, 5 min, 0.05\% trifluoracetic acid) and UV detection (254 nM). The purities of final compounds were $95 \%$ or greater. ${ }^{1} \mathrm{H}$ NMR spectra was recorded on Varian $500 \mathrm{MHz}$ operating at $500 \mathrm{MHz}$ for ${ }^{1} \mathrm{H} \mathrm{NMR}$ and ${ }^{13} \mathrm{C}$ NMR spectra was recorded on a Bruker NMR $400 \mathrm{MHz}$ Avance III spectrometer operating at $100 \mathrm{MHz}$ for ${ }^{13} \mathrm{C} \mathrm{NMR}$. Chemical shifts are given in part per million (ppm) relative to the deuterated solvent residual peak, coupling constants $J$ are given in Hertz.

2.1.1. General Procedure for the Synthesis of 3-Substituted-2-Cyano-2-Propenoic Acids

Step 1: Synthesis of 3-substituted-2-cyano-2-propenoic acid ethyl ester 
To a solution of aldehyde (1 equiv.) and ethylcyanoacetate (1.3 equiv.) in ethanol $(3 \mathrm{~mL})$ was added piperidine ( 0.2 quiv.) and the reaction mixture was stirred at $60{ }^{\circ} \mathrm{C}$ for $12 \mathrm{~h}$. The desired product crystallized out of solution upon cooling and was filtered and washed with $\mathrm{H}_{2} \mathrm{O}(2 \mathrm{x})$, a mixture of EtOAc/Hexanes (1:4) (2x) and dried under vacuum.

Step 2: Synthesis of 3-substituted-2-cyano-2-propenoic acids

Ethyl cycanoacrylate derivatives $(0.5 \mathrm{mmol})$ were dissolved in a $1: 1$ mixture of $0.5 \mathrm{~N}$ $\mathrm{LiOH}$ solution and THF overnight at room temperature. The THF solvent was removed under reduced pressure and a $2 \mathrm{~N}$ solution of $\mathrm{HCl}$ was added and the solution was stirred for $1 \mathrm{~h}$ at room temperature, resulting in the precipitation of white crystals. The resulting crystals were filtered with $\mathrm{H}_{2} \mathrm{O}(2 x)$ and a mixture of EtOAc/Hexanes (1:4) (2x) and were dried under vacuum to yield the final product in good yields.

\subsection{2. (E)-3-[5-(1,3-Benzothiazol-2-yl)-2-Furyl]-2-Cyanoacrylic Acid (BE1976)}

Yellow solid (87\%). ${ }^{1} \mathrm{H}$ NMR (500 MHz, acetone- $\left.d 6\right) \delta 98.25(\mathrm{dd}, J=8.2,1.2 \mathrm{~Hz}, 1 \mathrm{H})$, $8.17(\mathrm{~s}, 1 \mathrm{H}), 8.10(\mathrm{~d}, J=8.2 \mathrm{~Hz}, 1 \mathrm{H}), 7.64(\mathrm{q}, J=3.8 \mathrm{~Hz}, 2 \mathrm{H}), 7.60(\mathrm{ddd}, J=8.3,7.1,1.3 \mathrm{~Hz}$, $1 \mathrm{H}), 7.52$ (ddd, $J=8.3,7.2,1.2 \mathrm{~Hz}, 1 \mathrm{H}) ;{ }^{13} \mathrm{C}$ NMR (100 MHz, DMSO-d6) $\delta$ 164.04, 156.26, 154.10, 152.64, 150.29, 138.20, 135.34, 126.95, 126.27, 116.31, 114.70, 101.07. ESI-MS $(m / z)$ : $297(\mathrm{M}+1)^{+}$.

\subsection{3. (E)-2-Cyano-3-(1-Phenyl-4-Pyrazolyl)acrylic Acid (BE1978)}

White solid (52\%). ${ }^{1} \mathrm{H}$ NMR (500 MHz, acetone- $\left.d 6\right) \delta 9.06(\mathrm{~d}, J=2.2 \mathrm{~Hz}, 1 \mathrm{H}), 8.54(\mathrm{~d}$, $J=2.2 \mathrm{~Hz}, 1 \mathrm{H}), 8.40(\mathrm{~d}, J=2.2 \mathrm{~Hz}, 1 \mathrm{H}), 7.95(\mathrm{dd}, J=8.2,2.3 \mathrm{~Hz}, 2 \mathrm{H}), 7.63(\mathrm{td}, J=8.6,8.1,2.2$ $\mathrm{Hz}, 2 \mathrm{H}), 7.48(\mathrm{dd}, J=8.6,6.5 \mathrm{~Hz}, 1 \mathrm{H}) ;{ }^{13} \mathrm{C}$ NMR $(100 \mathrm{MHz}$, DMSO-d6) $\delta 163.91,145.99,141.81$, $138.95,132.86,130.27,128.27,119.73,117.90,117.26,100.80$. ESI-MS $(m / z): 240(\mathrm{M}+1)^{+}$.

\subsection{4. (E)-2-Cyano-3-(3,5-Dimethyl-1-Phenyl-4-Pyrazolyl)acrylic Acid (BE1980)}

White solid (74\%). ${ }^{1} \mathrm{H}$ NMR $(500 \mathrm{MHz}$, DMSO-d6) $\delta 9.06(\mathrm{~d}, J=2.2 \mathrm{~Hz}, 1 \mathrm{H}), 8.54(\mathrm{~d}$, $J=2.2 \mathrm{~Hz}, 1 \mathrm{H}), 8.40(\mathrm{~d}, J=2.2 \mathrm{~Hz}, 1 \mathrm{H}), 7.95(\mathrm{dd}, J=8.2,2.3 \mathrm{~Hz}, 2 \mathrm{H}), 7.63(\mathrm{td}, J=8.6,8.1$, $2.2 \mathrm{~Hz}, 2 \mathrm{H}), 7.48(\mathrm{dd}, J=8.6,6.5 \mathrm{~Hz}, 1 \mathrm{H}) 8.22(\mathrm{~s}, 1 \mathrm{H}), 7.62-7.42(\mathrm{~m}, 5 \mathrm{H}), 2.38(\mathrm{~s}, 3 \mathrm{H}), 2.35(\mathrm{~s}$, $3 \mathrm{H}) ;{ }^{13} \mathrm{C}$ NMR (100 MHz, DMSO-d6) $\delta 164.07,149.65,147.90,142.57,138.70,129.82,128.90$, $125.33,117.04,114.30,103.28,14.09,14.05$. ESI-MS $(m / z): 268(\mathrm{M}+1)^{+}$.

\subsection{5. (E)-2-Cyano-3-(p-Tolyl)acrylic Acid (BE1984)}

White solid (33\%). ${ }^{1} \mathrm{H}$ NMR $(500 \mathrm{MHz}$, acetone- $d 6) \delta 8.34(\mathrm{~s}, 1 \mathrm{H}), 8.09-7.98(\mathrm{~m}, 2 \mathrm{H})$, $7.47(\mathrm{~d}, J=8.1 \mathrm{~Hz}, 2 \mathrm{H}), 2.48(\mathrm{~s}, 3 \mathrm{H}) ;{ }^{13} \mathrm{C}$ NMR $(100 \mathrm{MHz}$, DMSO-d6) $\delta 163.72,154.62,144.30$, $130.24,130.19,116.56,102.64,21.64,21.59$. ESI-MS $(m / z): 186(\mathrm{M}+1)^{+}$.

\subsection{6. (E)-2-Cyano-3-(4-Phenyl-3-Pyrazolyl)acrylic Acid (BE 2617)}

Light brown solid (32\%). ${ }^{1} \mathrm{H}$ NMR $(500 \mathrm{MHz}, \mathrm{DMSO}-\mathrm{d} 6) \delta 8.58(\mathrm{~s}, 1 \mathrm{H}), 8.03(\mathrm{~d}$, $J=3.6 \mathrm{~Hz}, 1 \mathrm{H}), 7.72-7.33(\mathrm{~m}, 5 \mathrm{H}) ;{ }^{13} \mathrm{C}$ NMR $(100 \mathrm{MHz}, \mathrm{DMSO}-d 6) \delta 164.20,146.14,129.59$, 129.44, 117.39, 112.56, 99.53. ESI-MS ( $m / z): 240(\mathrm{M}+1)^{+}$.

\subsection{7. (E)-2-Cyano-3-[4-(p-Tolyl)-3-Pyrazolyl]acrylic Acid (BE 2623)}

White solid (57\%). ${ }^{1} \mathrm{H}$ NMR (500 MHz, DMSO-d6) $\delta 9.87(\mathrm{~d}, J=3.2 \mathrm{~Hz}, 1 \mathrm{H}), 8.01(\mathrm{~d}$, $J=3.0 \mathrm{~Hz}, 1 \mathrm{H}), 7.74-7.63(\mathrm{~m}, 2 \mathrm{H}), 7.51-7.18(\mathrm{~m}, 5 \mathrm{H}), 2.36(\mathrm{~d}, J=2.9 \mathrm{~Hz}, 3 \mathrm{H})$. ESI-MS $(m / z)$ : $254(\mathrm{M}+1)^{+}$. 


\subsection{Homology Modeling}

MPC1 and MPC2 monomers were modeled using the I-TASSER server based on the X-ray structures of bacterial SemiSWEET crystal structures [15-17]. I-TASSER uses a hierarchical approach to protein structure prediction and structure-based function annotation [18-20]. It first identifies structural templates from the PDB by the multiple threading approach, with full-length atomic models constructed by iterative template-based fragment assembly simulations. It was ranked as the top server for automated protein $3 \mathrm{D}$ structure prediction in recent CASP community-wide experiments for eight consecutive times. A model of the MPC dimer binding site was constructed by structural aliment using UCSF Chimera [21] based on the SemiSWEET transporter X-ray structure (PDB: 4X5M) as a template [16].

\subsection{Pharmacophore Modeling, Database Preparation, and Pharmacophore-Based Virtual Screening}

To develop a predictive model for a set of MPC inhibitors that can be used in virtual screening, we developed a pharmacophore model based on the chemical and geometrical features of UK-5099 using Phase [22]. The generated pharmacophore hypothesis is composed of three features: One negative charge $(\mathrm{N})$ corresponding to the carboxylic group, one hydrogen bond acceptor (A) corresponding to the cyano group and one aromatic ring $(\mathrm{Ar})$ corresponding to the indole ring. We then performed pharmacophore-based virtual screening of one million compounds from Enamine screening collection libraries to identify compounds that match the geometrical and chemical features represented by the pharmacophore hypothesis. The database was first processed by generating different protonation states, stereochemistry, tautomers, and ring conformations [23]. The relevant conformers (hits) were retrieved and aligned to the hypothesis. The hits were ranked by their PhaseScreenScore (fitness score), which is a score that measures how well the matching vector features (acceptors, donors, aromatic rings) overlay those of the hypothesis, and how well the matching conformation superimposes, in an overall sense, with the reference ligand conformation [24]. The fitness score was defined by:

$$
\mathrm{S}=\mathrm{W}_{\text {site }}\left(1-\mathrm{S}_{\text {align }} / \mathrm{C}_{\text {align }}\right)+\mathrm{W}_{\mathrm{vec}} \mathrm{S}_{\mathrm{vec}}+\mathrm{W}_{\mathrm{vol}} \mathrm{S}_{\mathrm{vol}}+\mathrm{W}_{\text {ivol }} \mathrm{S}_{\text {ivol }}
$$

$\mathrm{S}_{\text {align }}$ was the alignment score: RMS deviation between the site point positions in the matching conformation and the site point positions in the hypothesis. All user-adjustable parameters were kept as default values. $C_{\text {align }}$; alignment cutoff, default value $=1.2 . \mathrm{W}_{\text {site; }}$; Weight of site score, default value $=1.0 . \mathrm{S}_{\mathrm{vec}}$ Vector score; average cosine between vector features in the matching conformation and the vector features in the reference conformation. $\mathrm{W}_{\text {vec }}$ Weight of vector score, default value 1.0. $\mathrm{S}_{\mathrm{vol}}$ Volume score: Ratio of the common volume occupied by the matching conformer and the reference conformer, to the total volume (the volume occupied by both). Volumes were computed using van der Waals models of all non-hydrogen atoms. $W_{\mathrm{vol}}$ Weight of volume score, default value $=1.0 . \mathrm{S}_{\mathrm{ivol}}$ Included volume score: Ratio of the volume overlap between the matching conformer and the included volumes (if present) to the total included volume. Volumes were computed using van der Waals models of all atoms, except nonpolar hydrogens. $\mathrm{W}_{\mathrm{ivol}}$ Weight of volume score, default value $=0.0$.

\subsection{Bioluminescent Resonance Energy Transfer (BRET)-Based Assays for Inhibitor Binding}

Based on the success of a previously described MPC BRET reporter assay [25], we developed our own BRET assay using more recently adopted BRET donor and acceptor pairs. In this assay, Nanoluciferase is used as the BRET donor, and mCherry fluorescent protein is used as the BRET acceptor. Human MPC1 and MPC2 cDNA constructs with stop codon removed for C-terminal tagging were obtained from the Harvard PlasmID repository. MPC2 was cloned into pcDNA3.1-cccdB-Nanoluc obtained from Addgene (87067) by Gateway cloning; MPC1 was cloned into pDest-mCherry-N1, obtained from 
Addgene (31907), also by Gateway cloning. Site-directed mutagenesis (Agilent) was then performed to delete linking regions between the MPC proteins and C-terminal tags.

For BRET assays, U2OS cells were transiently transfected with MPC1-mCherry and MPC2-NLuc constructs and, $24 \mathrm{~h}$ later, plated onto 9 columns of a clear-bottom white 96-well plate at 10,000 cells/well. As a BRET control, some cells were transfected with MPC2-NLuc only, and these cells plated on the remaining 3 columns of the 96-well plate at 10,000 cells/well. The following day, cells were washed with PBS and starved in phenol red-, glucose-, and pyruvate-free Dulbecco's Modified Eagle's Medium (DMEM) for $3 \mathrm{~h}$. Cells were then washed in PBS and then provided PBS with $1 \mathrm{mM} \mathrm{CaCl}$ and $0.5 \mathrm{mM} \mathrm{MgCl} 2$ plus $2 \mu \mathrm{g} / \mathrm{mL}$ coelenterazine $\mathrm{h}$ BRET substrate, a white sticker applied to the bottom of the plate, and the plate immediately placed into a BioTek Synergy plate reader. Luminescence was then read repeatedly from the top of the plate at emission wavelengths of $485 / 20 \mathrm{~nm}$ and $640 / 40 \mathrm{~nm}$ for NLuc and mCherry signals, respectively. After several baseline readings, the plate was ejected, and test substrates/compounds were manually pipetted into the wells. All assays contained dimethyl sulfoxide (DMSO) vehicle-treated cells, as well as $5 \mu \mathrm{M}$ UK-5099 positive control cells. Pyruvate was treated at $5 \mathrm{mM}$, and all other test compounds were dosed at $10 \mu \mathrm{M}$. To calculate BRET activity, at each reading interval, the luminescence of $620 \mathrm{~nm}$ emission wavelength was divided by the $485 \mathrm{~nm}$ emission wavelength, and the values from the MPC2-Nluc-only expressing cells were subtracted from the cells expressing both constructs. All data was then normalized to the values immediately prior to compound injection. Data were either reported as curves over time or average normalized BRET of the several readings immediately after compound injections. For testing pyruvate/inhibitor-binding residues, point mutations were made in MPC1 or MPC2 constructs by site-directed mutagenesis (Agilent); BRET assays were performed the same as above. All of these assays contained 96-well plates directly comparing cells expressing WT MPC and mutant BRET constructs.

\subsection{Experimental Animals}

Care and use of C57Bl/6J mice conformed to standards established by the National Institutes of Health, approved by the IACUC of Saint Louis University \#2845. Mice were housed, up to 5 mice per cage, in ventilated cages and provided ad libitum access to standard rodent chow (5053 LabDiet) and water. Temperature and humidity of animal rooms was continuously monitored; rooms were set to a $12 \mathrm{~h}$ light/dark cycle at 6:00 AM-PM. Male and female mice were used for the described ex vivo experiments, at an age range of 8-20 weeks of age. For mitochondria isolation, mice were euthanized by $\mathrm{CO}_{2}$ asphyxiation. For primary hepatocyte isolation, mice were anesthetized with $2 \%$ isoflurane in $100 \%$ oxygen, and then euthanized by cervical dislocation. Additional mitochondrial respiration experiments were performed in mitochondria isolated from cardiac-specific MPC2-/ - mice, which were generated and described previously [26].

\subsection{Mitochondrial Isolation and Respiration}

Hearts were removed from mice after $\mathrm{CO}_{2}$ asphyxiation and homogenized in buffer containing $250 \mathrm{mM}$ sucrose, $10 \mathrm{mM}$ Tris base, and $1 \mathrm{mM}$ EDTA $(\mathrm{pH}=7.4)$ by $8-10$ passes of a glass-on-glass Dounce homogenizer on ice. Homogenates were centrifuged at $1000 \times g$ for $5 \mathrm{~min}$ at $4{ }^{\circ} \mathrm{C}$ to pellet nuclei and undisrupted cells. The supernatants were then centrifuged at $10,000 \times \mathrm{g}$ for $10 \mathrm{~min}$ at $4{ }^{\circ} \mathrm{C}$ to enrich for mitochondria; this mitochondrial pellet was washed and re-pelleted twice in fresh sucrose/tris buffer. The mitochondrial pellet was then solubilized in $\sim 150 \mu \mathrm{L}$ of Mir05 respiration buffer ( $0.5 \mathrm{mM}$ EGTA, $3 \mathrm{mM}$ $\mathrm{MgCl}_{2}, 60 \mathrm{mM}$ lactobionic acid, $20 \mathrm{mM}$ taurine, $10 \mathrm{mM} \mathrm{KH}_{2} \mathrm{PO}_{4}, 20 \mathrm{mM}$ HEPES, $110 \mathrm{mM}$ sucrose and $1 \mathrm{~g} / \mathrm{L}$ of fatty acid free bovine serum albumin; $\mathrm{pH}$ 7.1). Mitochondrial protein content was then measured by BCA; $50 \mu \mathrm{g}$ of mitochondrial protein was added to the chambers of an Oxygraph O2K (Oroboros Instruments), with a total volume of $2 \mathrm{~mL}$ Mir05 buffer set to $37^{\circ} \mathrm{C}$. Respiration was stimulated with $5 \mathrm{mM}$ pyruvate $/ 2 \mathrm{mM}$ malate and $2 \mathrm{mM}$ ADP. After obtaining steady state respiration measurements, compounds were 
added to the chamber at the indicated concentrations. Then, $5 \mathrm{mM}$ succinate was added to determine inhibitor specificity towards pyruvate-stimulated respiration. Steady-state rates of oxygen consumption were assessed for 1-2 min before addition of subsequent substrate or inhibitor. Oxygen consumption rates (OCR) were calculated from the change in oxygen concentration over time and normalized to $50 \mu \mathrm{g}$ of mitochondria within the chamber. For the experiments shown, respiratory control ratios (RCRs) were $\sim 70$, indicating high quality mitochondrial preparations.

\subsection{Primary Murine Hepatocyte Isolation and Culture}

Primary murine hepatocytes were isolated by perfusion of the liver with $1 \mathrm{mg} / \mathrm{mL}$ collagenase through the portal vein as previously described [5]. Cells were counted and plated onto 6-cm collagen-coated dishes in high glucose DMEM $+10 \%$ fetal bovine serum (FBS), Pen/Strep, and amphotericin B overnight. The following day, cells were treated with media + DMSO vehicle or $10 \mu \mathrm{M}$ MPC inhibitors for $20 \mathrm{~min}$. Cells were then scraped and collected into protein lysate buffer ( $15 \mathrm{mM} \mathrm{NaCl}, 25 \mathrm{mM}$ Tris Base, $1 \mathrm{mM}$ EDTA, 0.2\% NP$40,10 \%$ glycerol) with $1 \times$ protease inhibitor cocktail (cOmplete, Roche) and phosphatase inhibitors ( $1 \mathrm{mM} \mathrm{Na}_{3} \mathrm{VO}_{4}, 1 \mathrm{mM} \mathrm{NaF}$, and $1 \mathrm{mM}$ PMSF).

\subsection{Western Blotting Procedures}

Protein concentrations were determined using a Pierce MicroBCA kit (ThemoFisher), and $40 \mu \mathrm{g}$ of protein was loaded into the lanes of precast criterion $4-15 \%$ tris-glycine gels (Bio-Rad) and electrophoresed. Proteins were then transferred to Immobilon PVDF membranes (MilliporeSigma). Membranes were blocked with 5\% BSA in TBS-T for at least $1 \mathrm{~h}$ at room temperature. Primary antibodies were used at 1:1000 dilution in 5\% BSA-TBS-T overnight; then, membranes were washed $3 \times$ in TBS-T and subjected to LiCor near-IR secondary antibodies at 1:10,000 dilution in 5\% BSA-TBS-T for $1 \mathrm{~h}$. After washing $3 \times$ in TBS-T, membranes were developed using a LiCor Odyssey imaging system. Antibodies used were anti-mCherry (Cell Signaling 43590S), anti-MPC2 (Cell Signaling 46141S), antiphosphorylated PDH E1 $\alpha$ S232, S293, and S300 (MilliporeSigma AP1062, AP1063, AP1064), anti-PDH Cocktail (Abcam ab110416), and anti- $\alpha$ Tubulin (Sigma T5168); secondary antibodies used were anti-rabbit or anti-mouse IRDYE donkey 800CW (LiCor 926-32213 or 92632212). Full, un-cropped western blot images are provided in Supplemental Figure S1.

\subsection{Statistical Analyses}

All data are presented as mean \pm standard error of the mean, with statistical significance defined as $p<0.05$. Data were analyzed by one- or two-way ANOVA as appropriate, using GraphPad Prism version 9.3.1. Post hoc analysis was performed using Tukey's multiple comparison tests.

\section{Results}

\subsection{Homology Modeling of the MPC and Mutagenesis Studies}

The hMPC1/hMPC2 heterodimer has a greater binding efficiency for substrate and inhibitors than homodimers; the physiological relevance of MPC homodimers is not clear, due to the lack of stability of homodimers composed of endogenous proteins [1-7]. In order to map the substrate binding site and to gain understanding of the amino acid residues involved in substrate binding, we performed homology modeling of the heterodimer of MPC1 and MPC2 using I-TASSER (Iterative Threading Assembly Refinement) [18-20]. The server reported the AtSWEET13 sugar transporter (PDB:5XPD) to have the closest structural similarity for both monomers [15]. The dimer was constructed in the inward facing position based on structural alignment with the SemiSWEET transporter X-ray structure (PDB: 4X5M) as a template [16] (Figure 2A,B). During our work on this manuscript, Xu, et al., published a homology model of the MPC dimer and a molecular dynamics simulations study on the model [27]. Comparison of our homology model with the published model revealed high similarity. We identified potential substrate binding site residues and 
validated them using mutagenesis experiments. Structural alignments with other homolog transporters (SemiSWEET transporter PDB: 4QND and AtSWEET13 sugar transporter PDB: $5 \mathrm{XPD})$ predicted the location of the substrate binding cavity as the central cavity formed between both monomers (Figure 2C) [16,17].

A

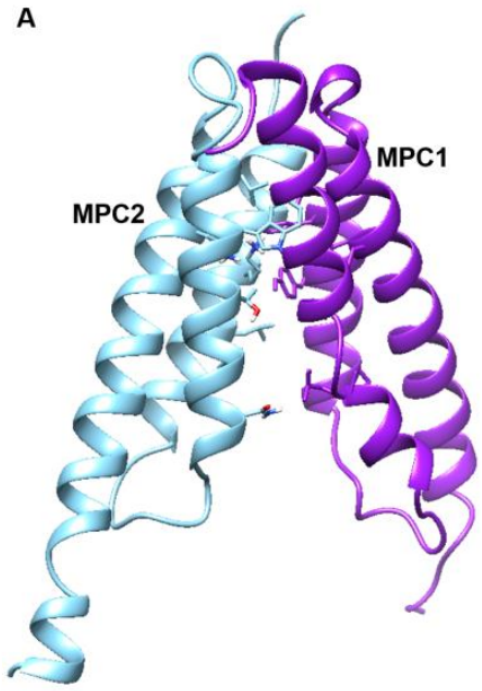

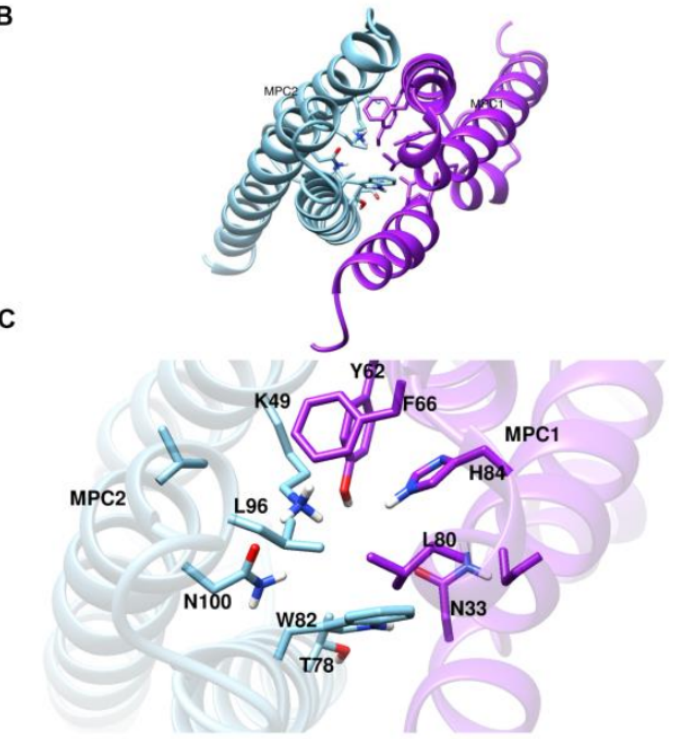

Figure 2. (A) Model structure of the mitochondrial pyruvate carrier $1 / 2(\mathrm{MPC} 1 / 2)$ heterodimer. (B) Cytosolic view of the binding pocket. (C) Residues constituting the putative substrate binding pocket shown as sticks.

To investigate the functional role of this putative substrate-binding pocket, we used site-directed mutagenesis to change MPC1 residues Asn33, His84 and Phe66 and MPC2 residues Trp82, Lys49 and Asn100 individually to alanine and assessed the effects of each of these mutations on the interaction with pyruvate or known MPC inhibitors using a bioluminescence resonance energy transfer (BRET)-based MPC reporter assay (Figure 3). In this system, MPC2 protein is C-terminally fused to Nanoluciferase (NLuc; photon donor), while MPC1 is C-terminally fused to mCherry (BRET acceptor). Pyruvate and MPC inhibitors induce a conformational change in the complex, reducing the distance between the donor and the acceptor, resulting in an increase in BRET signal. Mutation of MPC1 F66A, MPC2 K49A, or MPC2 N100A decreased the ability of pyruvate or inhibitors to increase the BRET signal, suggesting that the hydrophilic moiety of MPC2 Lys49 and Asn100 side chains and the hydrophobic aromatic side chain of MPC1 Phe66 are important for substrate or inhibitor binding (Figure 3A). This loss of BRET activity was not due to changes in MPC expression of these mutant constructs, as all mutants were well expressed (Figure 3B). These data further suggest that inhibitor and substrate binding to the MPC dimer involves hydrophobic or $\pi-\pi$ stacking interaction with the aromatic side chain of Phe66 and a salt bridge or hydrogen bonding interaction with the side chains of Lys49 and Asn100. 
A

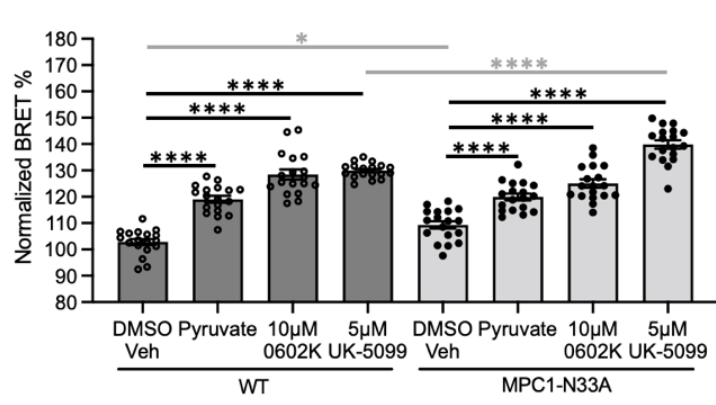

MPC1-H84A

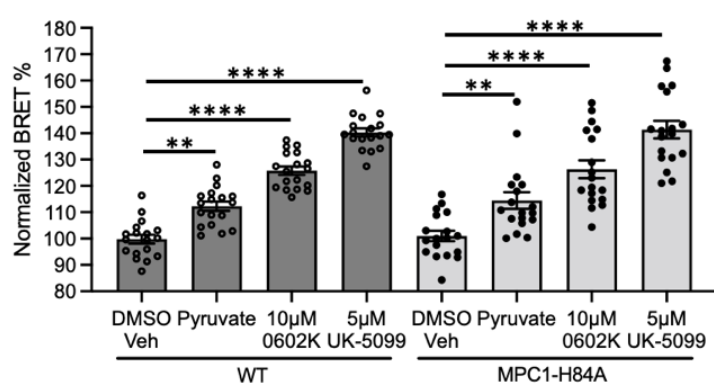

MPC2-W82A

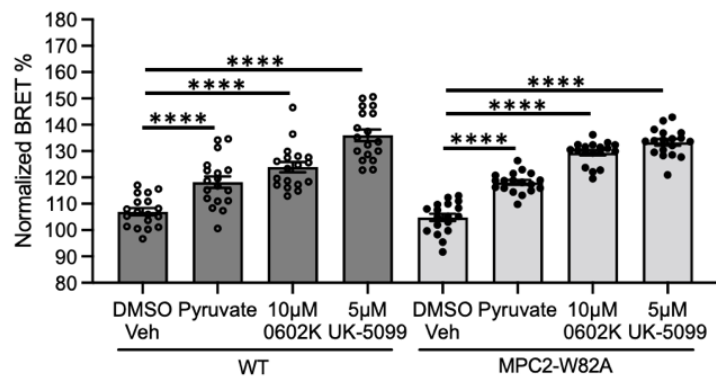

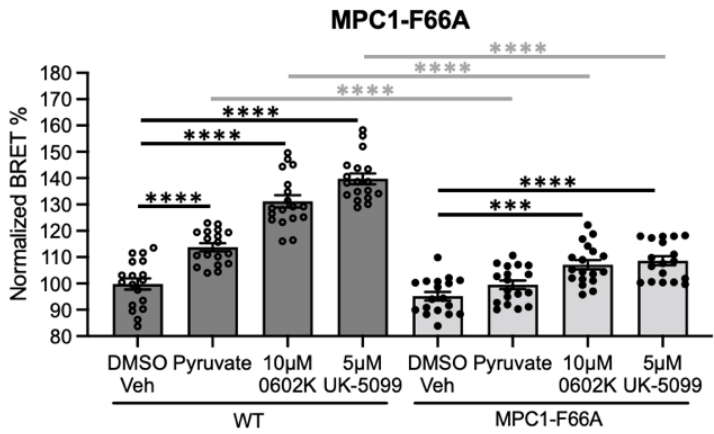

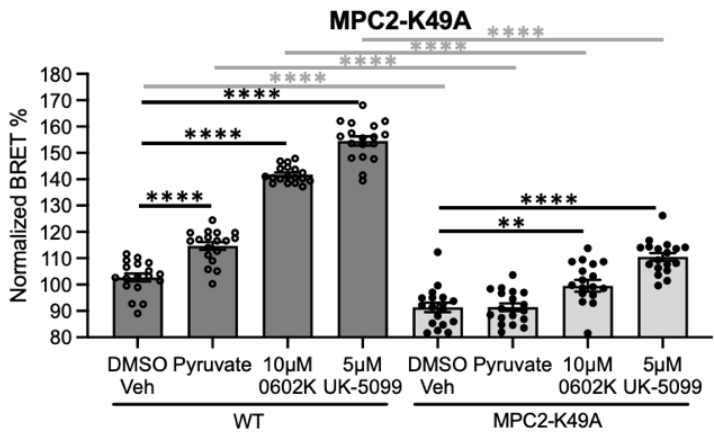

MPC2-N100A

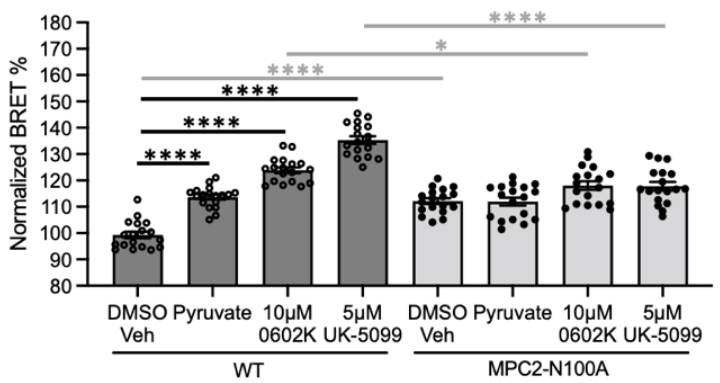

B

MPC1: - WT WT H84A WT WT F66A N33A

MPC2: - WT N100A WT W82A K49A WT WT

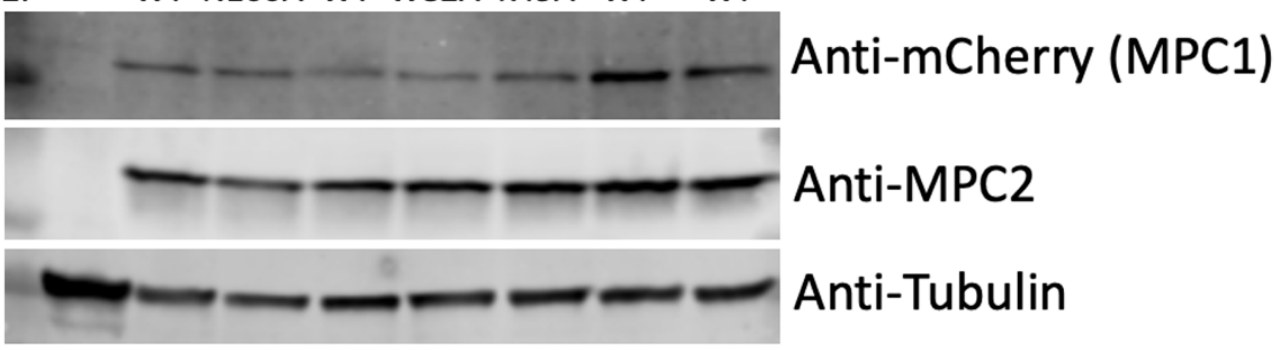

Figure 3. (A) Mitochondrial pyruvate carrier (MPC) bioluminescence resonance energy transfer (BRET) assay is activated by $5 \mathrm{mM}$ pyruvate or known MPC inhibitors ( $5 \mu \mathrm{M}$ UK-5099 or $10 \mu \mathrm{M}$ MSDC-0602K) compared to dimethyl sulfoxide (DMSO) vehicle controls. Mutation of MPC1-F66, MPC2-K49, or MPC2-N100 to alanine mostly abolishes the ability of pyruvate or inhibitors to increase BRET, suggesting mutation of these residues decreases binding and MPC conformational change. (B) Western blot images of U2OS cells transfected with WT or mutant MPC constructs indicates mutants are well expressed. Data presented as mean \pm S.E.M. Analyzed by two-way ANOVA with Tukey's correction for multiple comparisons ${ }^{*} p<0.05$, ${ }^{* *} p<0.01$, ${ }^{* * *} p<0.001$, ${ }^{* * * *} p<0.0001$; with black symbols for treatment comparisons and grey for genotype comparisons). 


\subsection{Pharmacophore Modeling}

To further identify inhibitor features responsible for ligand binding and to identify novel inhibitors, we developed a 3D-ligand-based pharmacophore hypothesis using Phase Suite [24]. These methods identify compounds that retain or match known features (acceptor, donor, negative ionic, positive ionic, hydrophobic, and aromatic ring) using known actives as a training set. Phase software generates pharmacophore hypotheses using a common pharmacophore perception algorithm based on conformational alignment of pharmacophore features. The hypothesis was built based on the structure of UK-5099, and is composed of three features: one negative charge $(\mathrm{N})$ corresponding to the carboxylic group, one hydrogen bond acceptor (A) corresponding to the cyano group and one aromatic ring (Ar) corresponding to the indole ring (Figure 4). This model is consistent with the homology modeling and mutagenesis data, which suggested the presence of $\pi-\pi$ stacking and two electrostatic interactions as important interactions for MPC inhibitor binding. Following the model generation, we conducted a pharmacophore-based virtual screen of over one million compounds from the Enamine advanced collection library. The screening collection was prepared and screened as discussed in the experimental section. To be classified as a hit, a compound was required to match all pharmacophore features of the pharmacophore hypothesis. The scoring function, PhaseScreenScore (Fitness score), was used to rank the screened compounds. The PhaseScreenScore measures how well the conformer matches the hypothesis and is a linear combination of the volume, site, and vector alignment scores. The top 7 scored compounds based on the PhaseScreenScore were selected for biological testing (Figure 5 \& Table 1). Two additional compounds (BE2617, BE2623) were synthesized based on structural similarity.

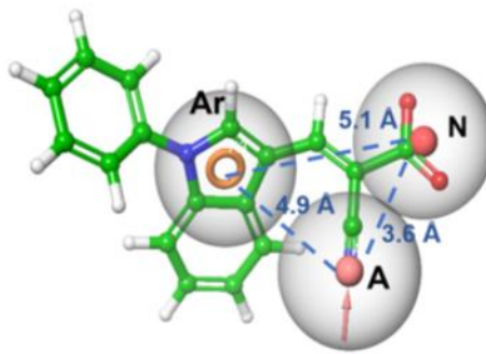

Pharmacophore modeling
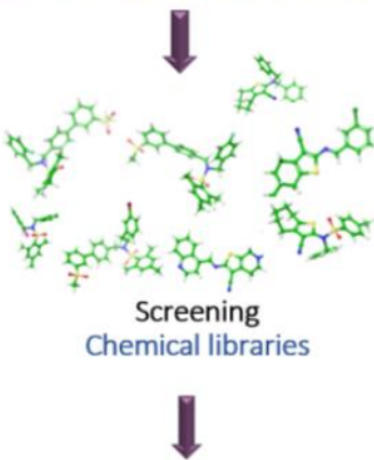

Scoring

Pharmacophore matching score

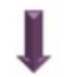

Compound selection \& in vitro assays

Figure 4. Pharmacophore modeling workflow for identification of novel mitochondrial pyruvate carrier (MPC) inhibitors. 
<smiles>O=C(NC(Cc1c[nH]c2ccccc12)C(=O)O)C1CC1</smiles><smiles>N#C/C(=C\c1ccc(-c2nc3ccccc3s2)o1)C(=O)O</smiles><smiles>N#C/C(=C\c1cnn(-c2ccccc2)c1)C(=O)O</smiles><smiles>Cc1nn(-c2ccccc2)c(C)c1/C=C(\C#N)C(=O)O</smiles>

BE1980<smiles>Cc1ccc(/C=C(\C#N)C(=O)O)cc1</smiles>

BE1984

inactive

$\mathrm{IC}_{50}(\mu \mathrm{M})=0.117$

$\mathrm{IC}_{50}(\mu \mathrm{M})=0.162$

$\mathrm{IC}_{50}(\mu \mathrm{M})=1.53$<smiles>CC(C)(C)c1ccc(C=C(C#N)C(=O)O)c(-c2cccc(Br)c2)n1</smiles>

$\mathrm{IC}_{50}(\mu \mathrm{M})=0.638$

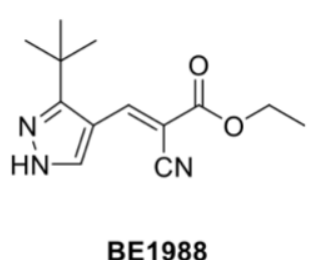

inactive<smiles>N#C/C(=C\c1n[nH]cc1-c1ccccc1)C(=O)O</smiles>

BE2617

$\mathrm{IC}_{50}(\mu \mathrm{M})=0.039$

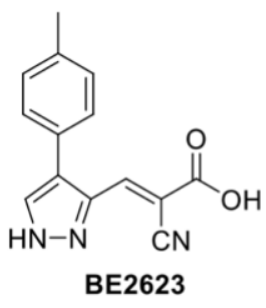

$\mathrm{IC}_{50}(\mu \mathrm{M})=0.731$

Figure 5. Hits selected for screening.

Table 1. PhaseScreenScore and relative $\mathrm{IC}_{50^{\prime}} \mathrm{s}$ of identified and synthesized mitochondrial pyruvate carrier (MPC) inhibitors.

\begin{tabular}{ccc}
\hline Compound & PhaseScreenScore & IC $_{\mathbf{5 0}}(\boldsymbol{\mu M})$ \\
\hline UK-5099 & 2.66 & 0.241 \\
BE1975 & 2.61 & inactive \\
BE1976 & 2.61 & 0.033 \\
BE1978 & 2.57 & 0.117 \\
BE1980 & 2.62 & 0.162 \\
BE1984 & 2.60 & 1.53 \\
BE1985 & 2.58 & 0.638 \\
BE1988 & 2.39 & inactive \\
BE2617 & N.D. & 0.039 \\
BE2623 & N.D. & 0.731 \\
\hline
\end{tabular}

N.D.: not determined.

\subsection{Identification and Validation of Novel MPC Inhibitors}

To validate the ability of identified compounds to inhibit MPC, we again used the BRET-based system that is sensitive to pharmacologic inhibitors of the MPC. We were delighted to find that five compounds (BE1976, BE1978, BE1980, BE1984, and BE1985) increased BRET activity, indicating a direct interaction (Figure 6A). The identification of these new non-indole inhibitors increases the chemical space around this scaffold and provides opportunity for drug design. Four of the five active compounds were synthesized in-house for further confirmation (see experimental section). Knoevenagel condensation of the appropriate aldehyde (1a-f) with 2-cyanoacetate (2) to form arylidenecyanoacetic acid ethyl ester (3a-f) followed by saponification using $\mathrm{LiOH}$ leads to the formation of BE1976, BE1978, BE1980, and BE1984 (Scheme 1). Two more derivatives BE2617 and BE2623 were synthesized and showed activity in the BRET assay. These two compounds were synthesized to explore the activity of $1 H$-pyrazol-3-yl as a core heterocycle vs. $1 H$ pyrazol-3-yl in BE1978, BE1980, and BE1985. 
A

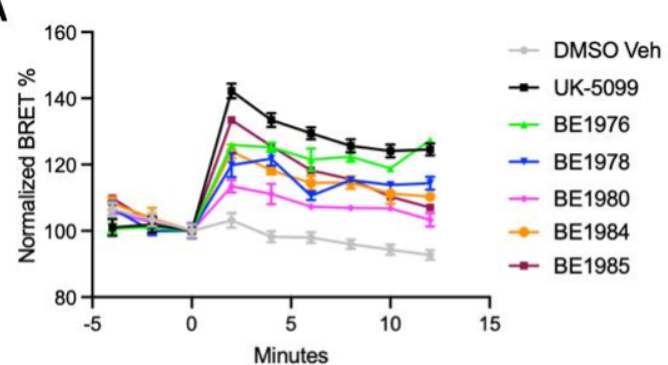

C

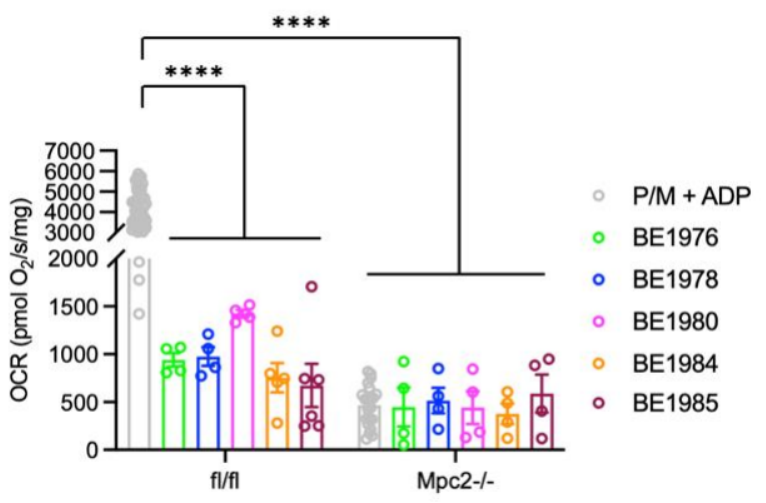

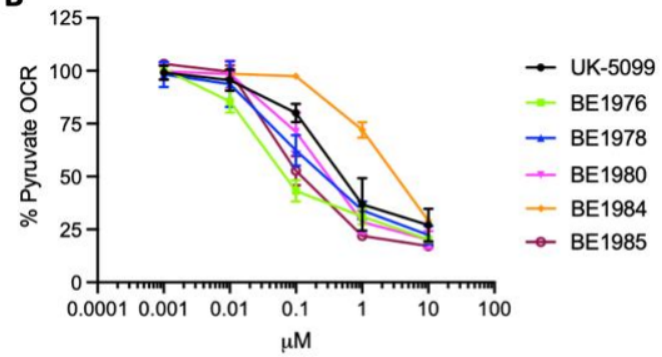
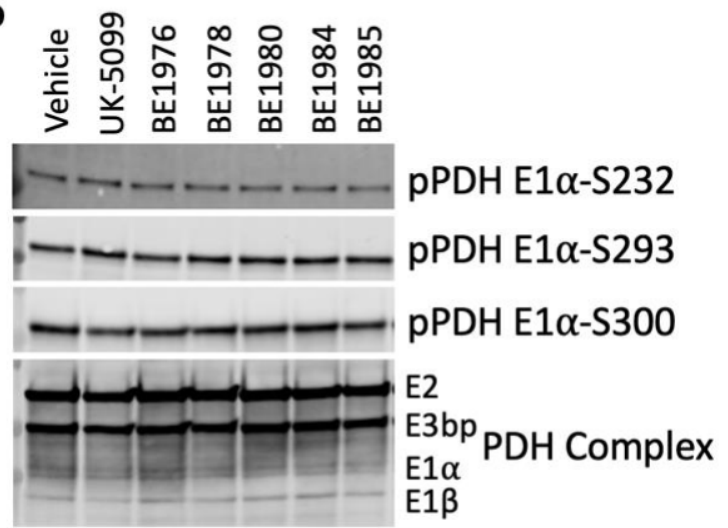

Tubulin

Figure 6. (A) Bioluminescent resonance energy transfer (BRET) activity in cells expressing WT mitochondrial pyruvate carrier (MPC) BRET constructs. Dimethyl sulfoxide (DMSO) vehicle or compound was added at time 0 . An increase is signal is indicative of binding and MPC conformational change. (B) Oxygen consumption rates (OCR) of isolated heart mitochondria with pyruvate, malate, and adenine diphosphate (ADP), in response to increasing concentrations of UK-5099, BE1976, BE1978, BE1980, BE1984, or BE1985. (C) OCR of WT and cardiac MPC2 - / - mitochondria respiring on pyruvate, malate and ADP without, and after addition of inhibitor compounds. (D) Western blot images of phosphorylated pyruvate dehydrogenase (PDH) E1 $\alpha$ and PDH complex in lysates from isolated primary hepatocytes after 20-min treatment of either DMSO vehicle or $10 \mu \mathrm{M}$ inhibitor compounds. Data presented as mean \pm S.E.M. Analyzed by two-way ANOVA with Tukey's correction for multiple comparisons $(* * * p<0.0001)$.

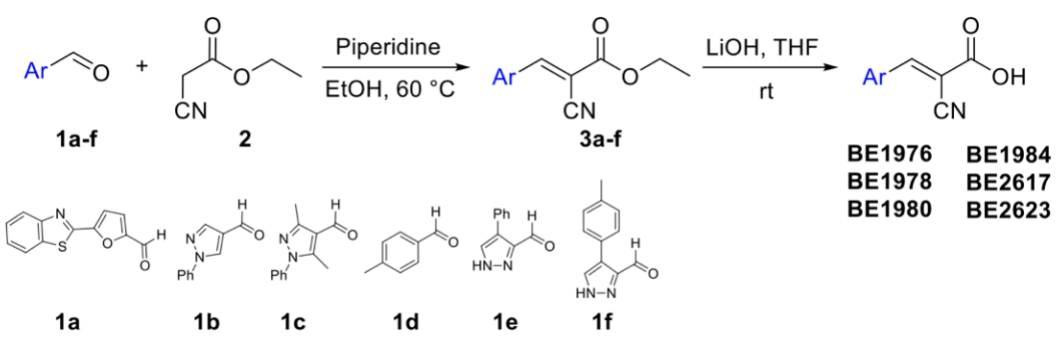

Scheme 1. Synthesis of identified mitochondrial pyruvate carrier (MPC) inhibitors.

Among the novel hits, BE1976 (2,5-substituted furan) and BE1978 (N-1 substituted pyrazole) showed the highest potency with an $\mathrm{IC}_{50}$ of $33 \mathrm{nM}$ and $117 \mathrm{nM}$, respectively, for inhibiting mitochondrial pyruvate respiration (Table 1). BE1976 was $>7$-fold more potent than UK-5099, while BE1978 was >2-fold more potent than UK-5099 (Table 1). The 3,5-dimethyl-1-phenyl pyrazole derivative BE1980 ( $\left.\mathrm{IC}_{50}=162 \mathrm{nM}\right)$ was a potent inhibitor but was weaker than BE1978, which showed that substitution at 3,5 positions of the pyrazole ring were tolerated but not favored. 3-Bromophenyl-1H-pyrazol cyanoacrylic acid BE1985 showed good inhibitory activity with an $\mathrm{IC}_{50}$ of $0.638 \mu \mathrm{M}$. BE1985 was 5- and 
4-fold less potent than the two corresponding N-1-substituted analogs BE1978 and BE1980, respectively (Table 1). The $p$-tolyl cyanoacrylic acid BE1984 showed weaker inhibition $\left(\mathrm{IC}_{50}=1.533 \mu \mathrm{M}\right)$ than the other heterocyclic analogs. Moreover, these compounds also inhibited mitochondrial respiration in a dose-dependent manner when pyruvate was provided as the respiratory substrate (Figure 6B). Additionally, these compounds did not reduce pyruvate respiration in $\mathrm{MPC} 2-/-$ cardiac mitochondria (Figure 6C) indicating MPC-dependent inhibition. Lastly, these compounds also did not increase the phosphorylation of pyruvate dehydrogenase (PDH) E1 $\alpha$, a proxy for decreased PDH activity, suggesting that these compounds decrease pyruvate oxidation by MPC inhibition and not by reduction of PDH activity (Figure 6D).

The two synthesized 1H-pyrazol-3-yl derivatives BE2617 and BE2623 were also found to be MPC inhibitors. Compound BE2617 was one of the most potent MPC inhibitors identified in this study, with an $\mathrm{IC}_{50}$ of 39 nM. BE2617 was comparable to BE1976 and was 6-fold more potent than UK-5099. The second derivative BE2623 $\left(\mathrm{IC}_{50}=0.731 \mu \mathrm{M}\right)$ was more than 18-fold less potent than BE2617. Interestingly, the minor structural difference between BE2617 and BE2623 resulted in a significant difference in activity, which warrants thorough SAR studies. Moreover, BE2623 was 3-fold less potent than UK-5099 in inhibiting the respiration and was comparable to the $1 H$-pyrazol-4-yl derivative BE1985 $\left(\mathrm{IC}_{50}=0.638 \mu \mathrm{M}\right)$. Elimination of the hydrogen acceptor (i.e., cyano group) as in BE1975 abolished activity. Similarly, esterification of the carboxyl group as in 3a-f and BE1988 rendered the compound inactive.

We also calculated a set of molecular descriptors to evaluate the drug-likeness of the identified inhibitors using QikProp program [23]. Identified compounds possessed drug-like properties and complied with Lipinski's Rule of Five. They were predicted to have good aqueous solubility, oral bioavailability, metabolic stability, and to be inactive in the central nervous system (Table 2). Human ether-a-go-go related gene (HERG) K+ channel blockers are potentially toxic; it is important to evaluate its inhibition as early as possible in drug discovery. The predicted $\mathrm{IC}_{50}$ values of our identified hits show that they are not predicted to cause cardiac toxicity. We will further test the in vitro absorption, distribution, metabolism, and excretion (ADME) of the best two lead compounds and optimize them accordingly for pharmacokinetics.

Table 2. Predicted absorption, distribution, metabolism, and excretion (ADME) of identified hits.

\begin{tabular}{|c|c|c|c|c|c|}
\hline Compound & $\mathrm{IC}_{50}(\mu \mathrm{M})$ & $c \log P$ & $\begin{array}{c}\text { HERG } \\
\log \text { IC }_{50}\end{array}$ & $\begin{array}{c}\text { \%Human Oral } \\
\text { Absorption }\end{array}$ & $\begin{array}{c}\log S_{\text {wat }} \\
(\mathrm{m} / \mathrm{L})(\text { Solubility) }\end{array}$ \\
\hline Range for $95 \%$ of Known Drugs & & $-2.0-6.5$ & $>-5$ & $>25 \%$ & $-6.5-0.5$ \\
\hline BE1976 & 0.033 & 2.37 & -3.879 & 70.347 & -4.728 \\
\hline BE1978 & 0.117 & 2.07 & -3.305 & 69.044 & -3.838 \\
\hline BE1980 & 0.162 & 3.05 & -3.209 & 78.955 & -5.05 \\
\hline
\end{tabular}

\section{Discussion}

The MPC complex is an attractive target for drug discovery, but the required properties for MPC inhibition and MPC complex composition are still unclear. The current study identified the putative substrate-binding cavity in the MPC dimer using homology modeling. Three amino acid residues Phe66 (MPC1) and Asn100 and Lys49 (MPC2) were validated by mutagenesis experiments to be important for substrate and/or inhibitor binding. While we showed that these mutant MPC1 or MPC2 proteins are individually expressed (Figure 3B), it is possible that these mutations affect the integrity of the MPC1/2 heterodimer. We do not suspect this for MPC1-F66A or MPC2-K49A, since there was still small, but significant, activation of BRET activity upon addition of MSDC-0602K or UK-5099 in these mutants (Figure 3A). However, the magnitude of these increases was much less than in WT-expressing cells. Interestingly, several of these mutants altered the basal BRET activity in the absence of pyruvate or inhibitor. MPC1-N33A and MPC2-N100A increased BRET, 
while MPC2-K49A decreased BRET signal (Figure 3A). This suggests that these mutations may change the MPC1/2 heterodimer structure sufficiently to alter the physical distance between the C-terminally-linked NLuc BRET donor and mCherry BRET acceptor. Overall, this study further validated and provided a promising computational approach for further investigations on the prediction of MPC inhibitors with pharmacophore modeling.

Five novel, non-indole MPC inhibitors were identified and validated experimentally, with two of them showing activity in the low nanomolar range (Table 1 and Figure 6). These compounds inhibited mitochondrial respiration in a dose-dependent manner but did not reduce pyruvate respiration in MPC2- / - cardiac mitochondria indicating MPC-dependent inhibition. The novel inhibitors decreased pyruvate oxidation by MPC inhibition and not through reduction in PDH activity. Because of the pronounced activity of BE1976 and BE2617, a more thorough characterization of its effects on pyruvate metabolism in cells and in vivo in future studies seems warranted. Additionally, a comprehensive structure-activity relationship study to optimize the pharmacokinetics of the novel inhibitors would provide important information for future drug discovery efforts aimed at inhibiting MPC activity.

The novel inhibitors described herein are structurally related to UK-5099 because they bear the $\alpha$-cyanoacrylic acid moiety. Although UK-5099 was identified as an MPC inhibitor in 1975 [12], there were no efforts to optimize this lead compound until 2021 when Liu and co-workers developed novel UK-5099 analogs as MPC inhibitors and used them topically to treat hair loss [11]. UK-5099 and the novel identified hits possess a Michael acceptor unit. Michael acceptors were historically excluded from drug discovery due to fear of off-target effects and potential toxicity. Recent success in developing targeted Michael acceptor drugs and the surge in number of FDA approved drugs and clinical candidates containing Michael acceptors, renewed the interest in this important category of compounds [28]. The identification of these new core scaffolds increases the chemical space around this scaffold and provides opportunity for drug design.

Inhibition of the MPC complex may be beneficial in a variety of chronic and progressive diseases. In some types of cancers, loss of MPC function has been related to cancer growth due to the role it plays in Warburg effect. Despite this, MPC inhibition has been established as a mechanism of action for lonidamine, an anti-tumor drug used to sensitize tumors to chemotherapy and radiotherapy [29], suggesting that mitochondrial pyruvate metabolism is important for cancer cell proliferation. MPC is also an attractive therapeutic target to treat neurodegenerative diseases linked to excitotoxicity [30]. Indeed, MPC inhibition protected primary cortical neurons from excitotoxic death without compromising energy metabolism, probably because of the metabolic flexibility of neurons [30]. Regulation of blood glucose concentrations and efficient hepatic gluconeogenesis require uptake and metabolism of pyruvate by mitochondria. Inhibition of the MPC in hepatocytes attenuates hyperglycemia in preclinical diabetes models; efficacy has also been demonstrated in clinical trials [5,6,31]. Moreover, growing scientific evidence suggests that targeting MPC is an effective strategy for the treatment of nonalcoholic fatty liver disease (NAFLD) and nonalcoholic steatohepatitis (NASH) [31,32]. A TZD MPC inhibitor, MSDC-0602, was shown to prevent liver fibrosis and reduce hepatic stellate cell activation in vitro [32]. Deletion of liver MPC2 in mice fed with trans-fatty acids rich diet protected them from developing NASH and reduced stellate cell activation [32]. Future studies will evaluate the effects of these novel inhibitors on relevant metabolic and pathogenic endpoints to determine their efficacy for treating diseases of interest.

\section{Conclusions}

Herein, we created a homology model of the MPC structure based upon similar protein structures for the bacterial SemiSWEET crystal structures. To validate this model structure, we performed site-directed mutagenesis and identified several amino acid residues that prevent the ability of a BRET based sensor (also newly developed in these studies) to bind pyruvate or known MPC inhibitors. We next generated a pharmacophore model that represent chemical and geometrical features of known MPC inhibitors and performed 
pharmacophore-based virtual compound screen of one million compounds library. We tested several of these screened chemicals in both BRET assays and in isolated mitochondrial respiration studies to validate MPC inhibition and determine relative $\mathrm{IC}_{50}$ concentrations for these novel inhibitors. We also describe that these inhibitors do not decrease pyruvate respiration in MPC $-/-$ mitochondria, nor do they alter phosphorylation of pyruvate dehydrogenase (a proxy of PDH activity); thus, they appear to specifically inhibit the MPC at low nanomolar concentrations. Lastly, we performed a predicted absorption, distribution, metabolism, and excretion (ADME) analysis on these compounds, which suggests good solubility and bioavailability. In future studies, we will advance these compounds to preclinical models of metabolic disease. But we believe this current work is a significant step forward in gaining understanding of the MPC structure and development of novel inhibitor compounds.

Supplementary Materials: The following supporting information can be downloaded at: https:// www.mdpi.com/article/10.3390/biomedicines10020365/s1, Figure S1: Uncropped western blot images.

Author Contributions: L.H., K.S.M., B.N.F. and B.E. designed the research, analyzed the data, and wrote the manuscript; L.H. and S.K. performed the computational studies, C.K. synthesized the compounds; L.E.G. performed the in vitro and ex vivo experiments, analyzed the data, and edited the manuscript; K.D.P. performed in vitro experiments and analyzed the data. All authors have read and agreed to the published version of the manuscript.

Funding: NIH R01 DK104735 supported work conducted in the laboratory of B.N.F., K.S.M is supported by NIH R00 HL136658.

Institutional Review Board Statement: Care and use of C57Bl/6J mice conformed to standards established by the National Institutes of Health and was approved by the IACUC of Saint Louis University protocol \#2845 (4/9/2019).

Informed Consent Statement: Not applicable.

Data Availability Statement: The datasets generated and/or analyzed in this study are available on reasonable request.

Acknowledgments: We would like to thank the Center for Clinical Pharmacology, Washington University School of Medicine and St. Louis College of Pharmacy, St. Louis, MO 63110, USA for funding provided to B.E.

Conflicts of Interest: B.N.F. is a shareholder and member of the scientific advisory board of Cirius Therapeutics, which is developing the MPC inhibitor MSDC-0602K for clinical use. The remaining authors have nothing to declare.

\section{References}

1. Herzig, S.; Raemy, E.; Montessuit, S.; Veuthey, J.-L.; Zamboni, N.; Westermann, B.; Kunji, E.R.S.; Martinou, J.-C. Identification and Functional Expression of the Mitochondrial Pyruvate Carrier. Science 2012, 337, 93-96. [CrossRef] [PubMed]

2. Bricker, D.K.; Taylor, E.B.; Schell, J.C.; Orsak, T.; Boutron, A.; Chen, Y.-C.; Cox, J.E.; Cardon, C.M.; Van Vranken, J.G.; Dephoure, N.; et al. A Mitochondrial Pyruvate Carrier Required for Pyruvate Uptake in Yeast, Drosophila, and Humans. Science 2012, 337, 96-100. [CrossRef] [PubMed]

3. Vigueira, P.A.; McCommis, K.S.; Schweitzer, G.G.; Remedi, M.S.; Chambers, K.T.; Fu, X.; McDonald, W.G.; Cole, S.L.; Colca, J.R.; Kletzien, R.F.; et al. Mitochondrial pyruvate carrier 2 hypomorphism in mice leads to defects in glucose-stimulated insulin secretion. Cell Rep. 2014, 7, 2042-2053. [CrossRef] [PubMed]

4. Bowman, C.E.; Zhao, L.; Hartung, T.; Wolfgang, M.J. Requirement for the Mitochondrial Pyruvate Carrier in Mammalian Development Revealed by a Hypomorphic Allelic Series. Mol. Cell. Biol. 2016, 36, 2089-2104. [CrossRef]

5. McCommis, K.S.; Chen, Z.; Fu, X.; McDonald, W.G.; Colca, J.R.; Kletzien, R.F.; Burgess, S.C.; Finck, B.N. Loss of Mitochondrial Pyruvate Carrier 2 in the Liver Leads to Defects in Gluconeogenesis and Compensation via Pyruvate-Alanine Cycling. Cell Metab. 2015, 22, 682-694. [CrossRef] [PubMed]

6. Gray, L.R.; Sultana, M.R.; Rauckhorst, A.J.; Oonthonpan, L.; Tompkins, S.C.; Sharma, A.; Fu, X.; Miao, R.; Pewa, A.D.; Brown, K.S.; et al. Hepatic Mitochondrial Pyruvate Carrier 1 Is Required for Efficient Regulation of Gluconeogenesis and Whole-Body Glucose Homeostasis. Cell Metab. 2015, 22, 669-681. [CrossRef] 
7. Rauckhorst, A.J.; Gray, L.R.; Sheldon, R.D.; Fu, X.; Pewa, A.D.; Feddersen, C.R.; Dupuy, A.J.; Gibson-Corley, K.N.; Cox, J.E.; Burgess, S.C.; et al. The mitochondrial pyruvate carrier mediates high fat diet-induced increases in hepatic TCA cycle capacity. Mol. Metab. 2017, 6, 1468-1479. [CrossRef] [PubMed]

8. Veliova, M.; Ferreira, C.M.; Benador, I.Y.; Jones, A.E.; Mahdaviani, K.; Brownstein, A.J.; Desousa, B.R.; Acín-Pérez, R.; Petcherski, A.; Assali, E.A.; et al. Blocking mitochondrial pyruvate import in brown adipocytes induces energy wasting via lipid cycling. EMBO Rep. 2020, 21, e49634. [CrossRef]

9. Panic, V.; Pearson, S.; Banks, J.; Tippetts, T.S.; Velasco-Silva, J.N.; Lee, S.; Simcox, J.; Geoghegan, G.; Bensard, C.L.; van Ry, T.; et al Mitochondrial pyruvate carrier is required for optimal brown fat thermogenesis. eLife 2020, 9, e52558. [CrossRef]

10. Sharma, A.; Oonthonpan, L.; Sheldon, R.D.; Rauckhorst, A.J.; Zhu, Z.; Tompkins, S.C.; Cho, K.; Grzesik, W.J.; Gray, L.R.; Scerbo, D.A.; et al. Impaired skeletal muscle mitochondrial pyruvate uptake rewires glucose metabolism to drive whole-body leanness. eLife 2019, 8, e45873. [CrossRef]

11. Liu, X.; Flores, A.A.; Situ, L.; Gu, W.; Ding, H.; Christofk, H.R.; Lowry, W.E.; Jung, M.E. Development of Novel Mitochondrial Pyruvate Carrier Inhibitors to Treat Hair Loss. J. Med. Chem. 2021, 64, 2046-2063. [CrossRef]

12. Halestrap, A.P. The mitochondrial pyruvate carrier. Kinetics and specificity for substrates and inhibitors. Biochem. J. 1975, 148, 85-96. [CrossRef]

13. Divakaruni, A.S.; Wiley, S.E.; Rogers, G.W.; Andreyev, A.Y.; Petrosyan, S.; Loviscach, M.; Wall, E.A.; Yadava, N.; Heuck, A.P.; Ferrick, D.A.; et al. Thiazolidinediones are acute, specific inhibitors of the mitochondrial pyruvate carrier. Proc. Natl. Acad. Sci. USA 2013, 110, 5422-5427. [CrossRef]

14. Hildyard, J.C.W.; Ämmälä, C.; Dukes, I.D.; Thomson, S.A.; Halestrap, A.P. Identification and characterisation of a new class of highly specific and potent inhibitors of the mitochondrial pyruvate carrier. Biochim. Biophys. Acta Bioenerg. 2005, 1707, 221-230. [CrossRef]

15. Han, L.; Zhu, Y.; Liu, M.; Zhou, Y.; Lu, G.; Lan, L.; Wang, X.; Zhao, Y.; Zhang, X.C. Molecular mechanism of substrate recognition and transport by the AtSWEET13 sugar transporter. Proc. Natl. Acad. Sci. USA 2017, 114, 10089-10094. [CrossRef]

16. Lee, Y.; Nishizawa, T.; Yamashita, K.; Ishitani, R.; Nureki, O. Structural basis for the facilitative diffusion mechanism by SemiSWEET transporter. Nat. Commun. 2015, 6, 6112. [CrossRef]

17. Xu, Y.; Tao, Y.; Cheung, L.S.; Fan, C.; Chen, L.-Q.; Xu, S.; Perry, K.; Frommer, W.B.; Feng, L. Structures of bacterial homologues of SWEET transporters in two distinct conformations. Nature 2014, 515, 448-452. [CrossRef]

18. Zhang, Y. I-TASSER server for protein 3D structure prediction. BMC Bioinform. 2008, 9, 40. [CrossRef]

19. Roy, A.; Kucukural, A.; Zhang, Y. I-TASSER: A unified platform for automated protein structure and function prediction. Nat. Protoc. 2010, 5, 725-738. [CrossRef]

20. Yang, J.; Yan, R.; Roy, A.; Xu, D.; Poisson, J.; Zhang, Y. The I-TASSER Suite: Protein structure and function prediction. Nat. Methods 2015, 12, 7-8. [CrossRef]

21. Pettersen, E.F.; Goddard, T.D.; Huang, C.C.; Couch, G.S.; Greenblatt, D.M.; Meng, E.C.; Ferrin, T.E. UCSF Chimera-A visualization system for exploratory research and analysis. J. Comput. Chem. 2004, 25, 1605-1612. [CrossRef] [PubMed]

22. Dixon, S.L.; Smondyrev, A.M.; Knoll, E.H.; Rao, S.N.; Shaw, D.E.; Friesner, R.A. PHASE: A new engine for pharmacophore perception, 3D QSAR model development, and 3D database screening: 1. Methodology and preliminary results. J. Comput. Aided. Mol. Des. 2006, 20, 647-671. [CrossRef] [PubMed]

23. Schrödinger Release 2020-1: QikProp; Schrödinger, LLC: New York, NY, USA, 2020.

24. Dixon, S.L.; Smondyrev, A.M.; Rao, S.N. PHASE: A novel approach to pharmacophore modeling and 3D database searching. Chem. Biol. Drug Des. 2006, 67, 370-372. [CrossRef] [PubMed]

25. Compan, V.; Pierredon, S.; Vanderperre, B.; Krznar, P.; Marchiq, I.; Zamboni, N.; Pouyssegur, J.; Martinou, J.C. Monitoring Mitochondrial Pyruvate Carrier Activity in Real Time Using a BRET-Based Biosensor: Investigation of the Warburg Effect. Mol. Cell 2015, 59, 491-501. [CrossRef]

26. McCommis, K.S.; Kovacs, A.; Weinheimer, C.J.; Shew, T.M.; Koves, T.R.; Ilkayeva, O.R.; Kamm, D.R.; Pyles, K.D.; King, M.T.; Veech, R.L.; et al. Nutritional modulation of heart failure in mitochondrial pyruvate carrier-deficient mice. Nat. Metab. 2020, 2, 1232-1247. [CrossRef] [PubMed]

27. Xu, L.; Phelix, C.F.; Chen, L.Y. Structural Insights into the Human Mitochondrial Pyruvate Carrier Complexes. J. Chem. Inf. Model. 2021, 61, 5614-5625. [CrossRef] [PubMed]

28. Ghosh, A.K.; Samanta, I.; Mondal, A.; Liu, W.R. Covalent Inhibition in Drug Discovery. ChemMedChem 2019, 14, 889-906. [CrossRef] [PubMed]

29. Nancolas, B.; Guo, L.; Zhou, R.; Nath, K.; Nelson, D.S.; Leeper, D.B.; Blair, I.A.; Glickson, J.D.; Halestrap, A.P. The anti-tumour agent lonidamine is a potent inhibitor of the mitochondrial pyruvate carrier and plasma membrane monocarboxylate transporters. Biochem. J. 2016, 473, 929-936. [CrossRef]

30. Divakaruni, A.S.; Wallace, M.; Buren, C.; Martyniuk, K.; Andreyev, A.Y.; Li, E.; Fields, J.A.; Cordes, T.; Reynolds, I.J.; Bloodgood, B.L.; et al. Inhibition of the mitochondrial pyruvate carrier protects from excitotoxic neuronal death. J. Cell Biol. 2017, 216, 1091-1105. [CrossRef] 
31. Harrison, S.A.; Alkhouri, N.; Davison, B.A.; Sanyal, A.; Edwards, C.; Colca, J.R.; Lee, B.H.; Loomba, R.; Cusi, K.; Kolterman, O.; et al. Insulin sensitizer MSDC-0602K in non-alcoholic steatohepatitis: A randomized, double-blind, placebo-controlled phase IIb study. J. Hepatol. 2020, 72, 613-626. [CrossRef]

32. McCommis, K.S.; Hodges, W.T.; Brunt, E.M.; Nalbantoglu, I.; McDonald, W.G.; Holley, C.; Fujiwara, H.; Schaffer, J.E.; Colca, J.R.; Finck, B.N. Targeting the mitochondrial pyruvate carrier attenuates fibrosis in a mouse model of nonalcoholic steatohepatitis. Hepatology 2017, 65, 1543-1556. [CrossRef] 